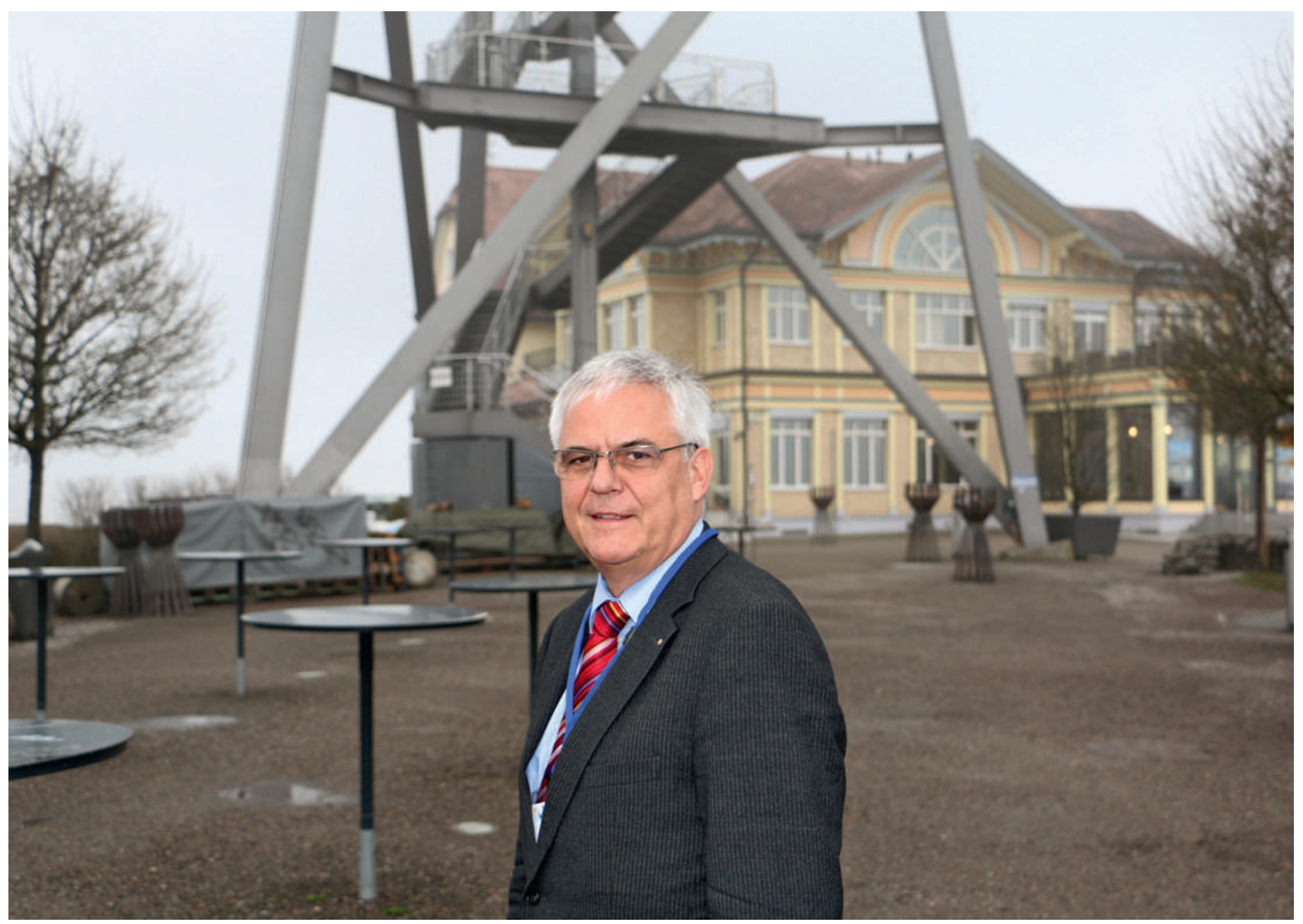

Neuer Gastgeber auf dem Uetliberg: Josef Widler, seit Anfang 2015 Präsident der Ärztegesellschaft des Kantons Zürich AGZ.

\title{
Im Zeichen der Kommunikation
}

\section{Bruno Kesseli (Text und Bilder)}

Dr. med. et lic. phil., Chefredaktor

«Kommunikation ist nicht alles, aber alles ist Kommunikation»: Zu diesem Thema boten die Zürcher Gesundheitstage der Ärztegesellschaft des Kantons Zürich AGZ auch bei der 9. Auflage ein anregendes Programm. Der neue AGZ-Präsident Josef Widler und sein Team hatten bei der Auswahl der Referentinnen und Referenten eine glückliche Hand.

«Never change a winning concept» könnte sich als Leitsatz beim Brainstorming des AGZ-Teams im Vorfeld der 9. Zürcher Gesundheitstage herauskristallisiert haben. Es gab für den neuen Präsidenten Josef Widler auch keinen Grund, die unter seinem Vorgänger Urs Stoffel etablierten Programmstrukturen grundsätzlich umzu- krempeln. Die Herausforderung bestand und besteht mit zunehmender Auflage des Symposiums vielmehr darin, dessen Frische zu erhalten, den Strukturen mit einem für das Publikum interessanten und relevanten Thema und spannenden Referentinnen und Referenten Leben einzuhauchen. Das ist auch diesmal wieder 


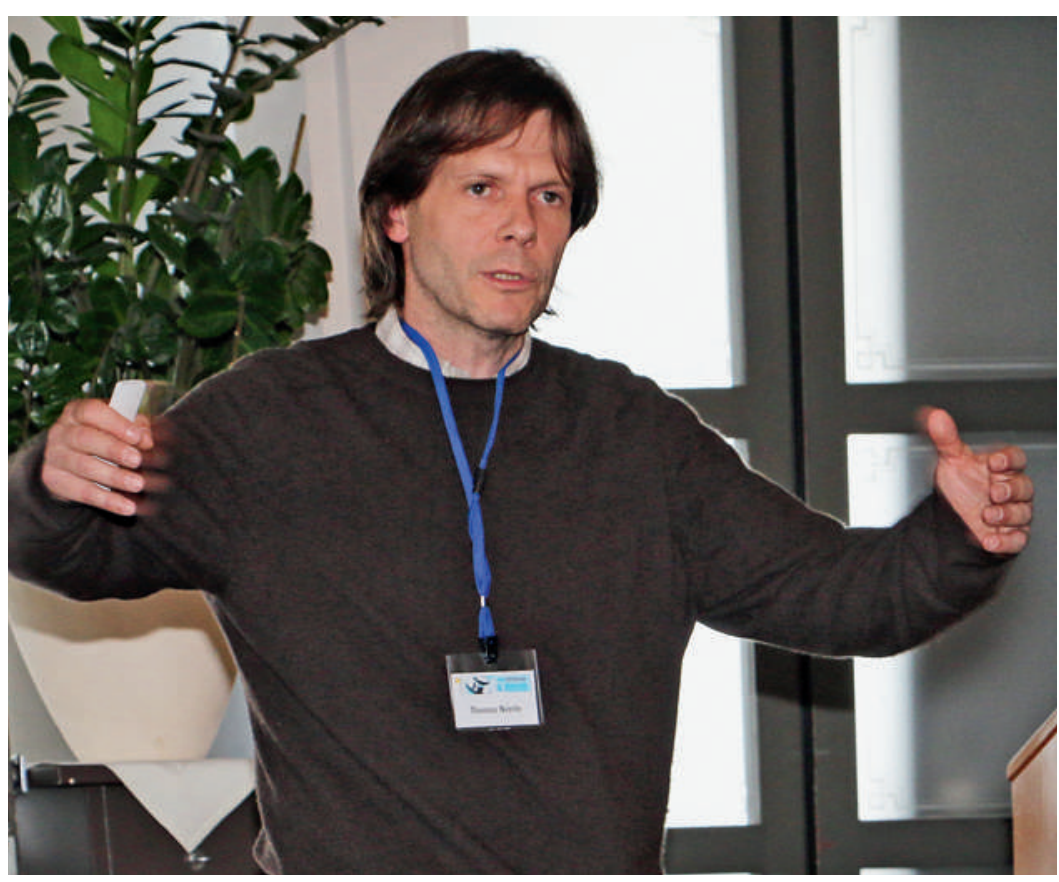

Thomas Nierle, Präsident von MSF Schweiz, sprach über Kommunikation in Krisenzeiten.

gelungen. «Kommunikation» ist thematisch zwar nicht gerade eine Neuentdeckung. Obwohl für den Arztberuf zentral, wurde sie aber in Aus-, Weiter- und Fortbildung lange Zeit eher stiefmütterlich behandelt, gerade was die Arzt-Patienten-Beziehung betrifft. Und in einer Zeit, in der sich die Kommunikationstechnologie in schwindelerregendem Tempo entwickelt, ist das Thema zweifellos von besonderer Aktualität.

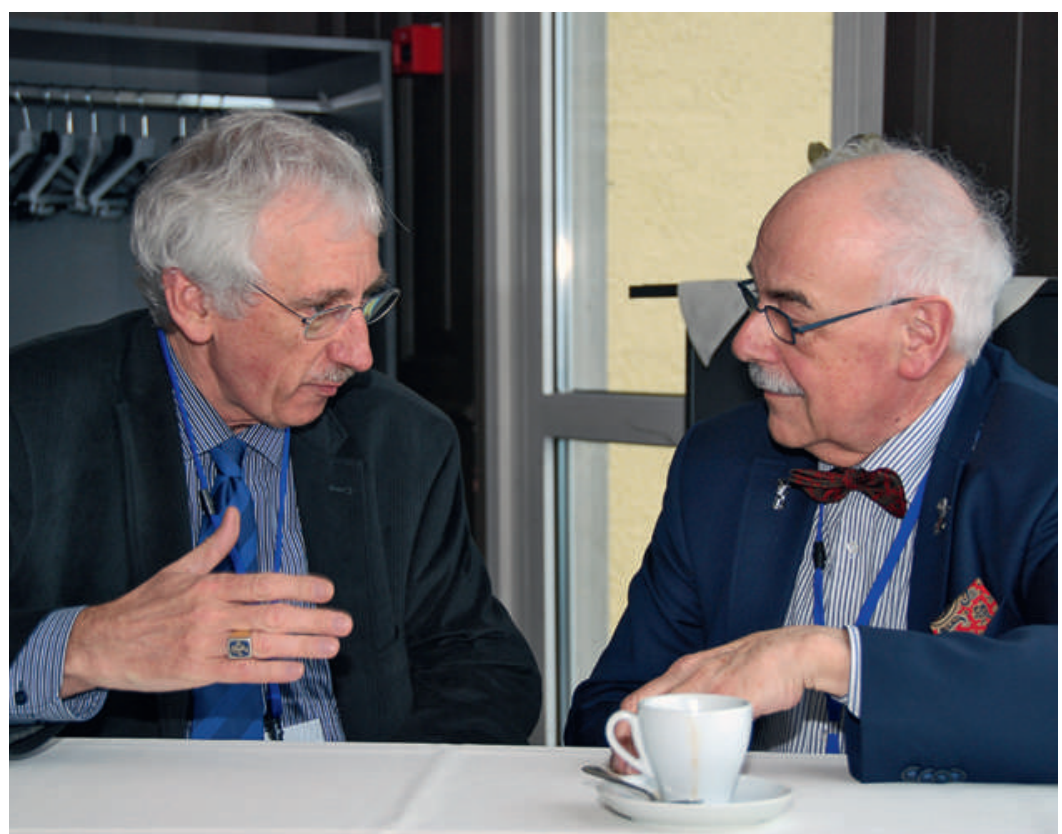

Die Uetliberg-Tagung bietet Raum für Gespräche: im Bild die Standespolitiker Peter Wiedersheim (links) und Christoph Ramstein. (Foto: AGZ)

\section{Sattelfester AGZ-Präsident}

Josef Widler bekam gleich zum Symposiumsauftakt reichlich Gelegenheit, sein Kommunikationstalent unter Beweis zu stellen, streikte doch die Technik, so dass seine Powerpoint-Folien partout nicht auf der Projektionsfläche erscheinen wollten. Eine Panne, die er gelassen meisterte, wobei ihm nicht nur seine Erfahrung als Arzt und Standespolitiker zugutegekommen sein dürfte. Auch als Politiker, der seit letztem Jahr dem Zürcher Kantonsparlament angehört und vor wenigen Wochen wiedergewählt wurde, wird er öfters zum Improvisieren gezwungen sein. Widler vermittelte den Eindruck eines pragmatischen, lösungsorientierten Arztes und (Standes-)Politikers, dem Bürokratie und Überreglementierung Dornen im Auge sind. Sowohl in der Arzt-Patienten-Beziehung als auch in der Zusammenarbeit zwischen der Ärzteschaft und anderen Gesundheitsberufen misst er dem gegenseitigen Vertrauen einen hohen Stellenwert bei. Für den Aufbau einer vertrauensbasierten Beziehung sei die Kommunikation zentral. "Ein Vertrauensverhältnis kann unter Umständen innert Sekunden entstehen», konstatierte der langjährige Hausarzt. Die Qualität der Kommunikation, so seine Erfahrung, sei oft entscheidend für die Qualität der gesamten Behandlung.

Auch beim Thema Qualität, dem er neben der Problematik des ärztlichen Nachwuchses einen kurzen standespolitischen Exkurs widmete, betonte er die Wichtigkeit des Vertrauens. Qualität verkomme im aktuellen Diskurs oft zu einer Worthülse, kritisierte er. «Wir müssen aufpassen, die Qualitätsbemühungen nicht zu pervertieren.» Dabei sprach er vor allem die ausufernde Kontrollbürokratie an, die ein Misstrauensverhältnis zwischen den Gesundheitsberufen fördere und zu ineffizienten Abläufen führe.

\section{Thomas Nierle: dynamisch und engagiert}

Von Effizienz und Misstrauen war auch im Referat von Thomas Nierle wiederholt die Rede. Der Internist, Chefarzt am Regionalspital Moutier und Präsident von Médecins Sans Frontières (MSF) Schweiz sprach am Beispiel der jüngsten Ebola-Epidemie in Westafrika über «Kommunikation in Krisenzeiten». Die Verhältnisse in den von Ebola am härtesten betroffenen Regionen brachten selbst die krisenerprobte medizinische Hilfsorganisation an ihre Limiten, auch in kommunikativer Hinsicht. Der Bevölkerung der Epidemiegebiete die Einsicht in die nötigen medizinischen und epidemiologischen Massnahmen zu vermitteln und ihr das Misstrauen gegenüber den bis zur Unkenntlichkeit vermummten, alienähnlichen Gestalten zu nehmen, 


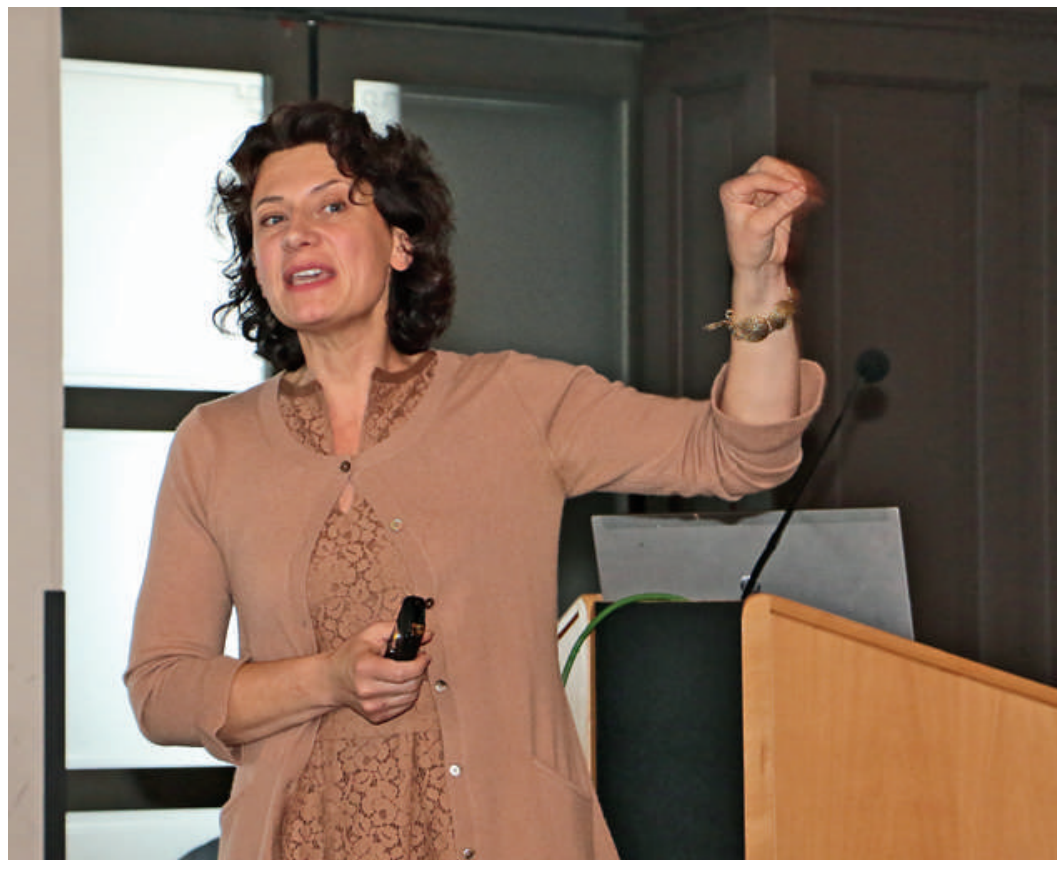

Charakterisierte die "Vernetzungsgesellschaft»: Andrea Belliger, Professorin an der Pädagogischen Hochschule Luzern.

die in ihren Wohngebieten auftauchten, gestaltete sich ebenso anspruchsvoll wie die Kommunikation mit Behörden, Regierungen und der WHO. Nierles engagierter Auftritt machte ohne Moralfinger deutlich, auf welch hohem Niveau wir hierzulande gelegentlich über die Unzulänglichkeiten unseres Gesundheitssystems jammern. Eindrücklich auch die Zahlen, die er zu MSF präsentierte. Für die 1971 gegründete Organisation sind mittlerweile rund 30000 Mitarbeiter tätig. MSF ist mit 372 Projekten in 72 Ländern präsent und hat ein Jahresbudget von 1,2 Milliarden Euro. Bemerkenswert ist, dass die Einnahmen zu über 85 Prozent von privaten Spendern stammen.

\section{Willkommen in der Vernetzungs- gesellschaft}

In eine ganz andere Welt entführte in der Folge Andrea Belliger das Publikum. Als Professorin und Prorektorin der Pädagogischen Hochschule Luzern und Co-Leiterin des Instituts für Kommunikation \& Führung IKF in Luzern forscht und lehrt sie zu Fragen von Trends und Veränderungen im gesellschaftlichen Kommunikationsverhalten. Sie machte an einer Vielzahl von Beispielen - auch und speziell aus dem Gesundheitsbereich deutlich, wie die rasanten medialen Entwicklungen der jüngeren Vergangenheit die Kommunikation nachhaltig verändert haben und weiter verändern. In der «Vernetzungsgesellschaft» stellt sich auch für Ärztinnen und Ärzte nicht primär die Frage, ob man die aktuellen Entwicklungen gut oder schlecht findet, sondern wie man damit umgeht.

\section{Von den "Ärzten der Demokratie» zur "(standes)politischen Lehrstunde"}

Wie breit das Spektrum auf dem Uetliberg war, mögen einige weitere Namen und Themen andeuten. Roger Köppel, Verleger und Chefredaktor der Weltwoche, präsentierte seine Sicht eines guten Journalismus und betonte, dass seine Metapher von den Journalisten als Ärzten der Demokratie durchaus ernst gemeint sei. Eva Gutmann, Biologin sowie Präsidentin der Kommission für soziale Sicherheit und Gesundheit (KSSG) des Zürcher Kantonsrats, und Thomas Kessler, Arzt und Leiter Neuro-Urologie der Universitätsklinik Balgrist, sprachen über Kommunikation in Tabu-Bereichen. Andreas Gattiker, Arzt und ehemaliger Vorsitzender der Geschäftsleitung GZO Spital Wetzikon, vermittelte am Beispiel seiner Institution Einblicke in die spezifischen Aspekte der Spitalkommunikation, die er zusammen mit der Swiss Aviation Training entwickelt hat. Für den philosophischen Touch war in gewohnt souveräner Manier der Philosoph und Publizist Ludwig Hasler besorgt. Und auch eine Art politische Elefantenrunde fehlte nicht: Auf einem Podium unter der Leitung von Iwan Rickenbacher gaben Josef Widler, der Zürcher Gesundheitsdirektor Thomas Heiniger und Nationalrätin Doris Fiala dem Publikum «eine (standes)politische Lehrstunde» in Sachen Kommunikation - so zumindest der als Podiumstitel formulierte Anspruch. 